\title{
Radiation Experience with the CDF Silicon Detectors
}

\author{
Ulrich Husemann \\ on behalf of the CDF Silicon and Radiation Monitoring Groups \\ University of Rochester, Rochester, New York 14627
}

\begin{abstract}
The silicon detectors of the CDF experiment at the Tevatron collider are operated in a harsh radiation environment. The lifetime of the silicon detectors is limited by radiation damage, and beam-related incidents are an additional risk. This article describes the impact of beam-related incidents on detector operation and the effects of radiation damage on electronics noise and the silicon sensors. From measurements of the depletion voltage as a function of the integrated luminosity, estimates of the silicon detector lifetime are derived.
\end{abstract}

Key words: $\mathrm{CDF}$, silicon detectors, radiation damage PACS: 29.40.Gx

\section{Introduction}

The CDF experiment [1] is a multi-purpose particle physics experiment at the Tevatron protonantiproton collider at Fermi National Accelerator Laboratory. At the Tevatron, protons and antiprotons are collided at a center-of-mass energy of $1.96 \mathrm{TeV}$. The CDF apparatus includes detectors for for calorimetry, particle identification, and tracking of charged particles in a 1.4 Tesla magnetic field. Tracking close to the interaction point and vertexing are performed by silicon micro-strip detectors. The CDF silicon detector system is comprised of three different detectors: Layer 00,

Email address: husemann@fnal.gov (Ulrich Husemann).

Preprint submitted to Elsevier Science
SVX II, and the Intermediate Silicon Layers (ISL). Layer 00 [2] consists of LHC-style single-sided silicon sensors mounted directly on the Tevatron beam-pipe to provide precision tracking close to the interaction point. The core detector for threedimensional tracking and vertexing is SVX II [3], built of five layers of double-sided silicon sensors. SVX II is used in the Silicon Vertex Trigger (SVT) [4], a trigger system sensitive to displaced vertices from the decays of long-lived particles. The ISL [5] link silicon tracks to the main drift chamber and extend the acceptance of the silicon detectors from $|z|=44 \mathrm{~cm}$ at a radius of $r \lesssim 10 \mathrm{~cm}$ (SVX II) to $|z|=90 \mathrm{~cm}$ at $r \lesssim 30 \mathrm{~cm}$. A summary of the detector properties and naming convention for Layer 00 and SVX II is given in Table 1. 
Table 1

Naming convention and detector properties for Layer 00 and SVX II.

\begin{tabular}{|c|c|c|c|c|}
\hline Layer & $\begin{array}{c}\text { Sensor } \\
\text { Manufacturer }\end{array}$ & $\begin{array}{c}\text { Strip } \\
\text { Orientation }\end{array}$ & $\begin{array}{c}\text { Radius } \\
(\mathrm{cm})\end{array}$ & $\begin{array}{c}\text { Max. } \\
\operatorname{Bias}(\mathrm{V})\end{array}$ \\
\hline Layer 00 & SGS-Thompson & axial & 1.35 & 500 \\
\hline Layer 00 & Hamamatsu & axial & 1.62 & 500 \\
\hline SVX L0 & Hama & $\operatorname{axial} / 90^{\circ}$ & 2.54 & 160 \\
\hline SVX L1 & Hamamatsu & axial $/ 90^{\circ}$ & 4.12 & 160 \\
\hline SVX L2 & Micron & $\mathrm{axial} / 1.2^{\circ}$ & 6.52 & 60 \\
\hline SVX L3 & Hamamatsu & $\mathrm{axial} / 90^{\circ}$ & 8.22 & 160 \\
\hline SVX L4 & Micron & axial $/-1.2^{\circ}$ & 10.10 & 60 \\
\hline
\end{tabular}

At the time of writing this article, the CDF silicon detectors have been irradiated with an integrated luminosity of $1.4 \mathrm{fb}^{-1}$, equivalent to a dose of approximately $3 \mathrm{kGy}$ from proton-antiproton collisions at the radius of SVX L0 [6]. The silicon detectors were originally designed to tolerate $2 \mathrm{fb}^{-1}$ to $3 \mathrm{fb}^{-1}$ before being replaced by a "Run IIb" upgrade. This upgrade was canceled, thus the silicon detectors must now operate to the end of Tevatron Run II in 2009 with an expected integrated luminosity of $4 \mathrm{fb}^{-1}$ to $8 \mathrm{fb}^{-1}$. Therefore, enhancing the detector longevity is a prime goal of the CDF silicon group.

\section{Beam-related Incidents}

High radiation levels due to uncontrolled beam losses of the Tevatron collider put the silicon detectors at risk. For example, 18 incidents in which the kicker magnets used to abort the Tevatron beam ramped spontaneously caused permanent damage in at least 26 of the 5644 readout chips. The CDF shift crew regularly evaluates potential risks for the silicon detectors based on systems to monitor the beam losses and the radiation dose close to the silicon detectors. In addition, an automated system triggers a beam abort if the dose rate is greater than $0.12 \mathrm{~Gy} / \mathrm{s}$. A prototype of a novel system to monitor and eventually abort the beam based on polycrystalline CVD diamond sensors has been successfully installed and tested in the CDF tracking volume. Fig. 1 shows a measurement of the diamond current during beam injection, acceleration, and collimation at the Tevatron.

The silicon detectors are powered by custom power supplies in CAEN SY527 mainframes. The

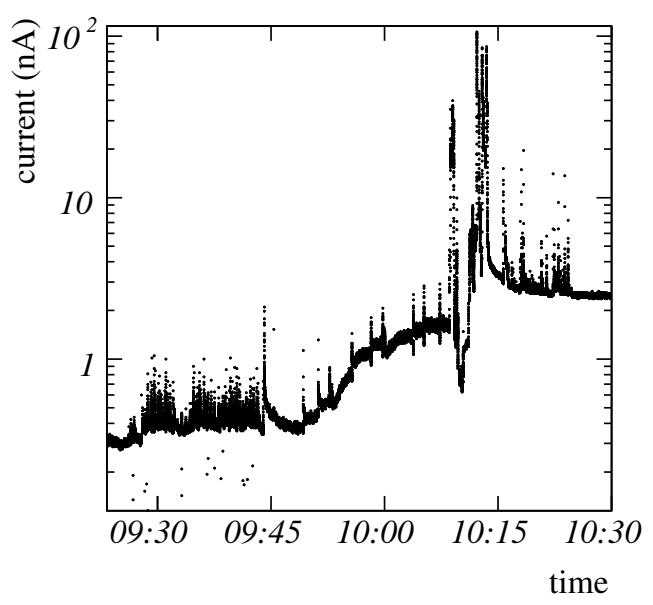

Fig. 1. Measured diamond current during Tevatron "shot setup". Beginning at 9:30, protons are injected, followed by nine antiproton injections after 9:45, and beam collimator positioning after 10:10.

power supplies are installed inside the CDF collision hall, at locations where no thick shielding can be installed. They show three common beamrelated non-permanent failure modes: spontaneous switch-offs, loss of communication, and corrupted read-back of currents and voltages. In 2005, the average rate of such failures was nine per month, and the failure rate of power supplies in areas of higher radiation was significantly larger than for power supplies in low-radiation areas [7].

\section{Radiation Damage}

The efforts to enhance the longevity of the CDF silicon detectors include measures to mitigate the effects of radiation damage and close monitoring of changes of the detector parameters under irradiation.

Radiation damage is mitigated by reducing the number of thermo-cycles of the system to a minimum and operating the silicon detectors at a lower temperature. The detector volume was thermally isolated in October 2004, and the detector temperature was lowered from $-6^{\circ} \mathrm{C}$ to $-10^{\circ} \mathrm{C}$ in early 2005.

A measure of the usable lifetime of the CDF silicon detectors is the lowest possible signal-to- 
noise ratio $(S / N)$ acceptable for the SVT and for the ability to perform efficient tagging of $b$ quark jets. Based on Monte Carlo simulations from CDF Run I, the lowest possible value is $S / N \approx 6$ [8]. The signal-to-noise ratio is determined by the noise level of sensors and readout electronics and by the charge collection efficiency of the sensors. Under irradiation, the noise level increases, and the charge collection efficiency decreases. A prediction of $S / N$ as a function of integrated luminosity can be found in [8].

Detailed tests have been performed to study the radiation hardness of the SVX3D readout chips and the optical transmitters used in the system. After $8 \mathrm{fb}^{-1}$ of integrated luminosity, an increase of $17 \%$ in the chip noise is expected for SVX L0 [9]. For the optical transmitters, a 10\% degradation of the light level is anticipated after $8 \mathrm{fb}^{-1}$; however, the waveform remains unchanged [10]. Therefore, neither the SVX3D chip nor the optical transmitters are expected to be the limiting factor for the silicon detector lifetime.

The dominant source of noise is the shot noise of the silicon sensors. The shot noise is increased by crystal defects in the sensors due to radiation damage. For SVX II, the shot noise can be parametrized as $Q=900 e \times \sqrt{I_{\text {leak }}}$, where $I_{\text {leak }}$ is the leakage current in $\mu \mathrm{A}[8]$. Changes in the leakage current can be approximated by changes in the bias current $I_{\text {bias }}$, assuming that all other contributions to the bias current are constant: $\Delta I_{\text {leak }} \approx \Delta I_{\text {bias }}$. An example of the evolution of $I_{\text {bias }}$ with integrated luminosity is shown in Fig. 2. The change in bias current is related to the fluence $\Phi$ by $\Delta I_{\text {bias }}=$ $\alpha \Phi V$, with the damage constant $\alpha$ and the known detector volume $V$. An effective damage constant of $\alpha_{\text {eff }}=(3.98 \pm 0.15) \times 10^{-17} \mathrm{~A} / \mathrm{cm}$ is derived by comparing the radial dependence of $\Delta I_{\text {bias }}$ with the radial dependence of the radiation field. The radiation field in the CDF tracking volume has been measured precisely by approximately 1000 thermal luminescent dosimeters (TLD) inside the CDF tracking volume [6]. The TLD measurements agree with measurements of $\Delta I_{\text {bias }}$ within $10 \%$ [11]. Hence, the fluence can be inferred from bias current measurements. The main systematic uncertainty of this method (13\%) is due to the imprecise knowledge of the sensor temperatures. From

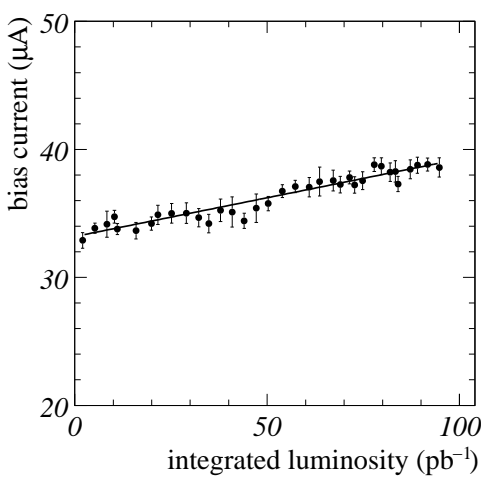

Fig. 2. Bias current $I_{\text {bias }}$ as a function of the integrated luminosity $\int \mathcal{L} \mathrm{d} t$ for a single silicon detector module in SVX II. The data points are compatible with a constant slope $\mathrm{d} I_{\text {bias }} / \mathrm{d} \int \mathcal{L} \mathrm{d} t$.

the measurement of the fluence, an update of the $S / N$ predictions as a function of luminosity in [8] will be derived.

Sufficient charge collection efficiency is achieved by applying a bias voltage between the two sides of a silicon sensor. Under irradiation and annealing, the effective number of charge carriers changes, and the bulk of the sensor undergoes "type inversion" from net $n$-type to $p$-type silicon. The bias voltage required to fully deplete the sensor decreases until type inversion occurs and increases again, eventually surpassing the maximum safe bias voltage. Breakdown of the coupling capacitors on the SVX II sensors limits the maximum safe bias voltage to approximately $160 \mathrm{~V}$ for the Hamamatsu sensors and to $60 \mathrm{~V}$ for the Micron sensors. The LHC-style sensors in Layer 00 can be biased up to $500 \mathrm{~V}$. In [8], a model of the depletion voltage is presented, and the lifetime of the silicon detectors is predicted, albeit with large uncertainties. To test the predictions of the lifetime model with data, the depletion voltage is measured on a regular basis in specialized "bias scan" runs [12]. For the singlesided sensors in Layer 00, the depletion voltage is determined from the charge collected from silicon hits on well-measured tracks as a function of the bias voltage. The double-sided sensors in SVX II allow for a measurement of the depletion voltage based on the reduction of inter-strip noise on the ohmic side of the sensor, without beam in the Tevatron. During the first $1.3 \mathrm{fb}^{-1}$ of integrated lumi- 


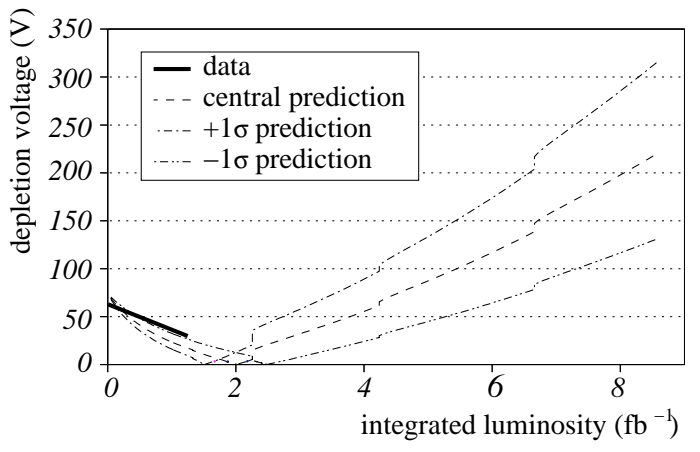

Fig. 3. Comparison of the depletion voltage $V_{\text {dep }}$ as a function of the integrated luminosity measured in bias scan runs (solid line) with a lifetime prediction for SVX L0 for $V_{\text {dep }}$ derived from the capacitance-voltage characteristic of the sensor [8] (central prediction: dashed line, $+1 \sigma$ prediction: dot-dashed line, $-1 \sigma$ prediction: dot-dot-dashed line).

nosity, 8 bias scans for Layer 00 and 13 bias scans for SVX II have been performed. The depletion voltage $V_{\text {dep }}$ as a function of integrated luminosity, is fitted with a straight line for every detector module and extrapolated to the point of type inversion. The results are consistent with the lifetime predictions presented in [8], as shown in Fig. 3. Layer 00 and SVX L0 are expected to undergo type inversion after $2 \mathrm{fb}^{-1}$ to $3 \mathrm{fb}^{-1}$. Assuming the same absolute value of the rate of change of $V_{\text {dep }}$ after type inversion, an upper limit of detector lifetime is estimated from the maximum safe bias voltage. Fig. 4 shows the predictions of the inversion point and the detector lifetime for SVX and Layer 00. Based on this estimate, the silicon detectors are expected to outlast Tevatron Run II; however, only a measurement of the rate of change of the depletion voltage after type inversion will lead to definite conclusions about the lifetime of the CDF silicon detectors.

This material is based upon work supported by the U.S. Department of Energy under Award Number DE-FG0291ER40685.

\section{References}

[1] A. Abulencia, et al., Measurements of inclusive $W$ and $Z$ cross sections in $p \bar{p}$ collisions at $\sqrt{s}=1.96 \mathrm{TeV}$, submitted to Phys. Rev. D.

[2] C. S. Hill, et al., Initial experience with the CDF Layer 00 silicon detector, Nucl. Instrum. Meth. A511

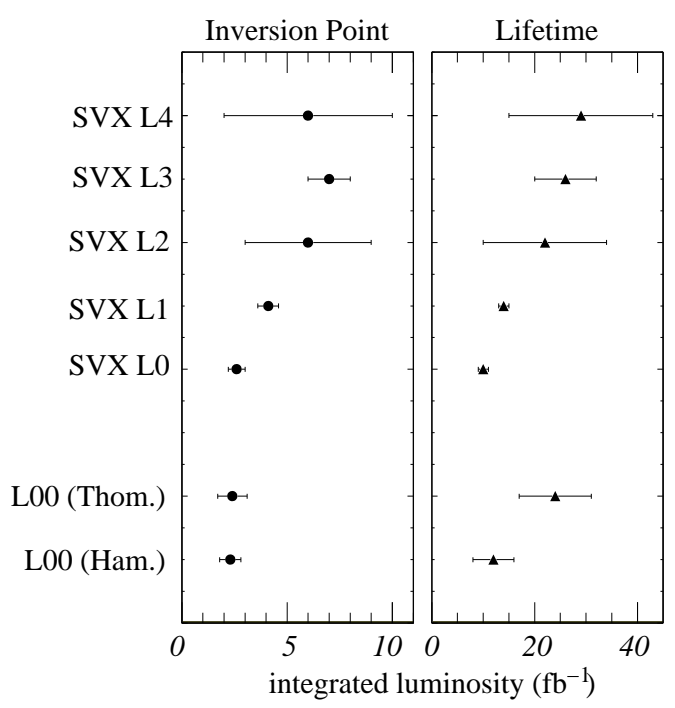

Fig. 4. Expected integrated luminosity for the point of type inversion (left) and lifetime (right) for the layers of SVX II and Layer 00 based on depletion voltage measurements [12].

(2003) 118-120.

[3] A. Sill, et al., CDF Run II silicon tracking projects, Nucl. Instrum. Meth. A447 (2000) 1-8.

[4] W. Ashmanskas, et al., The CDF silicon vertex trigger, Nucl. Instrum. Meth. A518 (2004) 532-536.

[5] A. Affolder, et al., Intermediate silicon layers detector for the CDF experiment, Nucl. Instrum. Meth. A453 (2000) 84-88.

[6] R. J. Tesarek, et al., A measurement of the radiation environment in the CDF tracking volume, Nucl. Instrum. Meth. A514 (2003) 188-193.

[7] M. Stanitzki, et al., Operational experience with the CDF Run II silicon detectors, Proc. 11th Workshop on Electronics for LHC and Future Experiments, Heidelberg, Germany, September 12-16, 2005.

[8] S. Worm, et al., Life expectancy of the CDF Run II silicon, Nucl. Instrum. Meth. A549 (2005) 126-129.

[9] M. Garcia-Sciveres, et al., The SVX3D integrated circuit for dead-timeless silicon strip readout, Nucl. Instrum. Meth. A435 (1999) 58-64.

[10] M. L. Chu, et al., Radiation hardness of the 1550-nm edge emitting laser for the optical links of the CDF silicon tracker, Nucl. Instrum. Meth. A541 (2005) 208212.

[11] S. D'Auria, et al., Measurement of the radiation field in CDF, Nucl. Instrum. Meth. A513 (2003) 89.

[12] A. Boveia, Status and performance of the CDF Run II silicon detector, PoS HEP2005 (2005) 377. 